education. In the course of the afternoon a lecture was delivered in the University Museum by Sir Arthur Smith Woodward on "Plot and Lhwyd and the Dawn of Geology". A survey was given of the position of natural science in Great Britain and in other European countries in the seventeenth century. The studies of Plot and Lhwyd were described. From the preface of the former to his work "The Natural History of Oxfordshire: an Essay towards the Natural History of England" (1677), it would appear that Plot was of vigorous temperament. He says : "But as for the hot-headed, half-witted Censurer, who, perhaps, only looks on the Title of a Chapter, or here and there a paragraph that makes for his turn, I must and do expect the lash of his tongue." The exhibition of objects of Ashmolean interestespecially those at the Bodleian Library in relation to Ashmole himself, and of great historic valuewere a welcome form of dedication accompanying the celebrations.

\section{The Neutron}

THE Bakerian lecture of the Royal Society was delivered on May 25 by Dr. J. Chadwick, who took as his subject "The Neutron". Neutrons can be liberated by the bombardment of several light elements by $\alpha$-particles, but, except from beryllium and boron, the yield of particles is very small. In some cases the experiments are consistent with the conservation of energy and momentum in the reactions. With beryllium, however, it is at first sight difficult to account for the whole of the energy available in the disintegration. From the data now available, the mass of the neutron is consistent with the view that the neutron is a complex particle formed by the union of a proton and an electron. Other arguments suggest that the neutron is an elementary particle. As an alternative, one might suppose the proton to be complex, consisting of a neutron and a positive electron, but this view also has certain difficulties. Dr. Chadwick dealt with experiments on the passage of neutrons through matter. Some interesting points appear in the collisions with the lighter nuclei, in particular with protons. In some cases inelastic collisions have been observed in which the atomic nucleus is disintegrated. When the radiation from beryllium, consisting of neutrons and $\gamma$-rays, passes through matter, positive electrons are occasionally produced. It is not yet known whether these are due to the action of the neutrons or to the $\gamma$-rays. The function of the neutron in the structure of atomic nuclei was also discussed.

\section{Crested Rat at the London Zoo}

The authorities at the gardens of the Zoological Society have been fortunate in securing four specimens, from Kenya, of that most curious and rare rodent the 'crested rat' (Lophiomys imhausi); no specimen of this animal has ever before been exhibited in captivity, though it has long been known to science, having been described by Milne-Edwards so long ago as 1867. 'Though termed as 'rat', it has really no very close relationship to this animal.
Indeed, owing to its many structural peculiarities it has been made to constitute a family by itselfthe Lophiomidæ. Nor is it at all accurately described when it is said to be a "badger-like animal", for it bears no very close resemblance to any other known rodent. One of its most characteristic external features is the great crest of long coarse hair which surmounts the back, bordered on each side by two bands of white. The nature and function of a glandular tract running down from the nape tail-wards, along the back, has yet to be discovered. Until now, nothing was known of the young. But one of the new captives, since its arrival, has given birth, not to a litter, as one would have expected of a rodent, but to a single young one, and this has elicited a further fact new to science, since it was born with a coat of fur, and not naked as with other rodents. The fact that this animal is arboreal is not likely to have any connexion with this unusual condition of the new born young, and the fact that but one is produced at a birth.

\section{Research in the Paint and Varnish Industries}

ON May 25 the Research Association of British Paint, Colour and Varnish Manufacturers held an exhibition at its Research Station, Waldegrave Road, Teddington, Middlesex. The firms comprising the Association's members range from those concerned with the manufacture of linoleum to the manufacturers of printing inks and of polishes, the main body conforming in interests with the title of the Association. In the six years that the Association has been actively engaged in researches, this wide range of interests has necessarily given rise to a large number of apparently unrelated investigations. The industries concerned, however, all have to deal with the properties of fluids, of thin films, of pigments and with the inter-relations between the pigment and an embedding medium. The work on view on May 25 included new apparatus and technique for the measurement of the hiding power of pigments, of the gloss of surface films and of the tinting, fastness and bronzing characteristics of pigments, the rate of evaporation of volatile constituents from thin films, the reactions of detached films of paints, varnishes and lacquers to a steady load, a rapidly increasing load, and an alternating load. New methods of evaluating the technical utility of linseed, tung and other vegetable oils were on view, as well as smallscale manufacturing plant suited to the production of all types of varnishes, lacquers and paints. The very rapid recent advances in the field of synthetic resins were reflected in a series of chemical studies of the mechanism involved in the complex polymerisation processes on which the manufacture of these materials is based. In the field of pigment manufacture, several important advances in technique recently originated at the Research Station were on view. These concern improvements in dispersion of organic lakes, greatly increased resistance of lead chromes to photo-chemical and chemical influences and the treatment of prussian blues to overcome some of their usual defects from the point of view of dispersibility. 